\title{
Modelo de generación de valor mediante el capital intelectual en empresas de base tecnológica de software
}

\author{
Model for generating value through intellectual capital in technology-based \\ software companies
}

\author{
Angela María Lanzas Duque* \\ Universidad Tecnológica de Pereira, Colombia
}

Recibido el 2 de febrero de 2018; aceptado el 24 de agosto de 2018

Disponible en Internet el: 6 de septiembre de 2019

\section{Resumen}

El objetivo de esta investigación es proponer un modelo que permitiera identificar si el capital intelectual (CI), compuesto del capital humano, del capital relacional y el capital estructural, impacta positivamente en la generación de valor de las empresas de base tecnológica (EBT) dedicadas al desarrollo de software. Para definir el modelo se estudió y analizó la teoría referente al CI y la generación de valor, sus componentes y la relación entre ellos; para obtener la información empírica de la investigación, se utilizó como instrumento una encuesta en línea, la cual tuvo en cuenta elementos de diferentes modelos de valoración de intangibles, los cuales se adaptaron de acuerdo a las características propias de las empresas objeto de estudio. Para contrastar las hipótesis, identificar la relación entre las variables y validar el modelo planteado se utilizó el análisis estadístico multivariado, mediante la técnica de modelos de ecuaciones estructurales (MEE), considerando en los diferentes análisis la significancia estadística y práctica.

Código JEL: D23, L86, G12, C39, C51

\footnotetext{
*Autor para correspondencia

Correo electrónico amlanzas@utp.edu.co (A.M. Lanzas Duque).

La revisión por pares es responsabilidad de la Universidad Nacional Autónoma de México. 
Palabras clave: Capital intelectual; Empresas de base tecnológica; Generación de valor; Modelos de ecuaciones estructurales

\begin{abstract}
The objective of this research is to propose a model to identify whether intellectual capital, composed of human capital, relational capital and structural capital, has a positive impact on the value of technology-based companies dedicated to software development. To define the model, we studied and analyzed the theory of intellectual capital and value generation, as the elements that compose it and the interrelation between them. To obtain the empirical information of the research, an online survey was used as instrument in order to construct the survey and define the indicators of the model, elements of different intangible valuation models were taken into account, which were adapted considering the characteristics of the companies under study.To test the hypotheses, to identify the relationship between the variables and to validate the proposed model, multivariate statistical analysis was used, using the Structural Equation Modeling (SEM) technique, considering in the different analyzes the statistical and practical significance.
\end{abstract}

JEL codes: D23, L86, G12, C39, C51

Keywords: Intellectual capital; Technology-based companies; Value generation; Structural equation models

\title{
Introducción
}

Con la aparición y desarrollo en las últimas décadas de la nueva economía, la economía del conocimiento y la información, las empresas han empezado a darle un papel protagónico al conocimiento, pues lo están considerando como elemento fundamental para la generación de valor, ventajas y riqueza. Este conocimiento está siendo trasformando en información, que las conlleve a construir nuevas formas, métodos y maneras de abordar los problemas para dar un valor agregado que sea útil para la sociedad. (Lanzas, A. 2016)

Este valor agregado que actualmente buscan dar las empresas a sus bienes, servicios, métodos, procesos y demás, las ha obligado a replantear y reestructurar la política tradicional de inversión en activos que se había dado en décadas pasadas, como era la inversión en capital tangible (máquinas, materias primas, entre otros), pasando a la inversión en capital intangible, también llamado capital intelectual, como son: satisfacción del cliente, capacidades de los trabajadores, marca, sistemas de información, know how, entre muchos otros. . (Lanzas, A. 2016)

Lo que más ha impulsado y contribuido con el desarrollo de esta nueva economía es la globalización, esto hizo que se ampliaran las fronteras y los mercados, y que la competencia empezara a ser global, obligando a los productores a ser más competitivos, a invertir mucho 
más en investigación y desarrollo, a innovar y a fortalecer y formar a sus colaboradores (Torres, 2012, p.17).

En el sentido de los colaboradores, Nevado y López (2004) afirman "que el desempeño de las sociedades actuales depende crecientemente de lo que logren hacer para preparar a su gente, desarrollar su capacidad de investigación e innovación, crear sistemas para acceder, guardar, procesar y usar información, acerca de la inversión en la formación de su CI’. (p.165)

Todo lo anterior ha llevado a que en Colombia (país donde se lleva a cabo la investigación), se hayan formulado e implementado en las últimas décadas políticas y estrategias encaminadas a fortalecer la creación, apropiación y trasferencia del conocimiento, como la Ley de Ciencia 1286 de 2009, la Ley de Emprendimiento 1014 de 2016, el Plan de Desarrollo Nacional de Colombia 2014-2018, entre otros; que buscan desarrollar estrategias para generar conocimiento e innovación en el aparato productivo e impregnar una cultura de innovación y emprendimiento en todas las esferas del Estado.

Las tendencias de la economía, los planes, leyes y programas anteriormente mencionados han propiciado los espacios y las condiciones para que en Colombia surjan un nuevo tipo de empresas llamadas empresas de base tecnológica (EBT), es decir empresas basadas en el conocimiento y la innovación.

Para apoyar a las EBT los diferentes actores de la sociedad en Colombia (Estado, empresa y universidad) de manera coherente e integrada han realizado proyectos y programas con el fin de que dichas empresas, comprendan, se integren y desarrollen estrategias para involucrarse de manera efectiva en la nueva sociedad de la información y la economía del conocimiento. Dichos apoyos se han centrado principalmente en proporcionar mecanismos de financiación (de hecho insuficientes) y propiciar espacios de trabajo junto con universidades, grupos de investigación, parques tecnológicos, incubadoras de empresas, entre otros, encaminados a fortalecer el desarrollo tecnológico; sin embargo, no acompañan a las empresas en sus procesos de desarrollo y gestión, de hecho se evidencia que en gran medida las EBT en Colombia, están presentando deficiencias en su gestión interna, pues han concentrado sus esfuerzos en el desarrollo técnico especializado de sus productos o servicios y han descuidado los procesos internos de tipo financiero y administrativo que les permiten determinar el impacto de sus decisiones (Padilla, 2015).

Es claro y de alguna forma lógico que las EBT dediquen la mayor parte de sus esfuerzos y recursos en el desarrollo y creación del conocimiento y la innovación, pero el estar descuidando y desatendiendo los procesos internos propios de las mismas, las puede llevar a perder oportunidades y a no generar valor para sus dueños.

Para realizar la investigación se tomaron las EBT pertenecientes al parque Tecnológico Parquesoft Colombia, las cuales son empresas dedicadas al desarrollo de soluciones informáticas con alto componente innovador. 


\section{Marco Teórico}

\section{Capital intelectual (CI); nuevo activo en la economía del conocimiento}

El concepto de $\mathrm{CI}$ ha sido tratado por diversos autores y no existe como tal una definición de aceptación general, pero si se evidencia en casi todas las definiciones elementos comunes que hacen referencia a creación de valor, activos intangibles (sin sustancia física), activos estratégicos, conocimiento y competencias, principalmente.

El Instituto Universitario Euroforum Escorial (1998), citado por Ochoa, M (2010) define el CI "como el conjunto de activos intangibles de una organización que, pese a no estar reflejados en los estados financieros tradicionales, en la actualidad, el mercado percibe que generan valor o tienen potencial de generarlo en el futuro." p.35.

Para Edvinsson y Malone (1998) el CI se refiere a las relaciones con los clientes y proveedores, al conocimiento que posee la empresa, a las capacidades tecnológicas, a la experticia de sus colaboradores, entre otros, capaces de generar una ventaja competitiva en el mercado.

Ordoñez, P. (2004) citando a Bueno, E (1998) define CI como "el conjunto de competencias básicas distintivas: unas, de origen tecnológico, en donde se incluyen también todos los elementos del saber y de la experiencia acumulados por la empresa; otras de origen organizativo o propias de los "procesos de acción" de la organización, y otras, de carácter personal, concernientes a las actitudes, aptitudes y habilidades de los miembros de la organización". p. 134

Para efectos de esta investigación se entenderá como CI todos aquellos activos que posee la empresa de tipo intangible, que se originan del conocimiento y que ofrecen posibilidades de generar valor si gestionan de manera adecuada y que no aparecen registrados en los estados financieros.

\section{Componentes del Capital Intelectual (CI)}

Para esta investigación se revisaron varias propuestas de clasificación presentadas por diversos autores y se decidió tomar la clasificación propuesta por Sveiby (2007), la cual se aproxima a las clasificaciones dadas por Edvinsson y Malone (1998), Kaplan y Norton (2008), Nevado y López (2013), Brooking (1996), Modelo Meritum, Camisón, Lapiedra, Segarra y Boronat, (2003) (Modelo Nova) y Modelo Intelect, de igual forma se validó dicha clasificación con las características propias de las empresas objeto de estudio; por lo tanto, el CI está constituido por los siguientes componentes:

- Capital humano (CH): El capital humano se centra como su nombre lo indica en las personas que hacen parte de la organización, se incluye el trabajo en equipo, sistemas de remuneración e incentivos, cultura para la innovación y programas de formación y capacitación.

- Capital estructural (CE): Es el conocimiento que la empresa apropia y que le genera 
valor, el cual permanece en el tiempo. Comprende las rutinas organizativas como sistemas de información y comunicación, procesos de investigación y desarrollo, y estructuras organizacionales flexibles.

- Capital relacional (CR): Se refiere al valor que tiene para una empresa el conjunto de relaciones que mantiene con el medio, ya sea con sus clientes, proveedores o socios de I+D y la percepción que estos tienen de ella. Incluye trabajo en equipos con clientes, relación con proveedores, trabajo con grupos de investigación y participación en redes,

\section{Empresas de Base Tecnológica (EBT) - Parquesoft Colombia}

Se puede definir como EBT aquellas organizaciones generadoras de valor mediante la aplicación sistemática de conocimientos tecnológicos y científicos, las cuales diseñan, desarrollan y elaboran productos, servicios, procesos y procedimientos con altos de componentes de innovación. (Colciencias, 2008).

Para Fariñas y López (2006), citado por Merritt, H (2012), la creación de EBT presenta grandes ventajas económicas y sociales debido a su potencial para lograr altas tasas de crecimiento en un plazo relativamente corto, generar artículos innovadores, crear empleos de alta calidad, además de su proclividad para generar productos con un mayor valor agregado y mejores tasas de ganancia. (Lanzas, A. 2016)

Las empresas objeto de estudio pertenecen a una fundación colombiana sin ánimo de lucro que se fundó en el año 1999, cuyo propósito es facilitar a jóvenes emprendedores la creación y desarrollo de EBT que proveen al mercado productos y servicios de tecnología informática, llamado Parquesoft Colombia. Las empresas del parque tienen las siguientes características (Lanzas, A. 2016):

- $\quad$ Surgen de iniciativas emprendedoras en universidades, colegios e institutos.

- Funcionan los primeros años como empresas informales, se constituyen legalmente en el momento en que tienen formalizados sus procesos y tienen cierto posicionamiento en el mercado. En los primeros años las negociaciones las realizan en cabeza del Parque.

- Son empresas donde principalmente los colaboradores son sus dueños; recurren a contratación de terceros en la medida que desarrollan proyectos específicos.

- Trabajan en redes asociativas para satisfacer las necesidades de los clientes, una empresa apoya a la otra en el momento de satisfacer las necesidades de los clientes.

- Son fuertes en los procesos de desarrollo de software, pero carecen de procesos administrativos y financieros bien definidos.

- En su gran mayoría se encuentran ubicadas físicamente en las instalaciones que ofrece el Parque, este cubre los gastos de conectividad, arriendo, servicios públicos y demás.

- Tienen fuerte relaciones con grupos de investigación y sectores gubernamentales, para desarrollar procesos de investigación y obtener recursos de financiamiento. 


\section{Generación de valor}

El objetivo tradicional de las empresas ha sido conseguir el máximo beneficio con el fin de lograr su supervivencia, actualmente las organizaciones tienen como nuevo fin crear o generar valor, no solo para supervivir, sino también para crecer en el tiempo.

Generar valor es un proceso que consiste en identificar, crear y mantener oportunidades y conocimiento con el fin de permitir a las empresas obtener ventajas para sostenerse y crecer en el corto, mediano y sobretodo largo plazo; esto requiere que se integren de manera sistémica los objetivos corporativos con las estrategias y recursos necesarios para alcanzarlos. Al amparo de este proceso las empresas pueden ser orientadas, administradas y medidas, siempre con el fin de crear valor.

La creación de valor supone identificar y aprovechar los recursos valiosos que tiene la empresa con el fin de mejorar su efectividad y esto se vea traducido en beneficios tanto para el cliente, como para sus colaboradores y sobretodo sus dueños; también supone a la empresa el poder superar las expectativas iniciales y que esto le posibilite tener elementos diferenciadores que le generen ventajas en el mercado.

"Por valor entendemos aquella cualidad convencional del objeto que no es atribuida de un cálculo o una peritación. El valor no es un hecho sino una opinión” Álvarez (2010) citando a Brilman (1990).

Tradicionalmente cuando se habla de resultados de valor y creación de valor en las empresas, esto se asocia con indicadores de tipo económico y financiero como rentabilidad, liquidez, riesgo, estructura financiera; para medir la gestión de la empresa en su proceso de creación de valor se utilizan herramientas de tipo financiero como son rentabilidad económica (ROI), rentabilidad financiera (ROE), valor económico agregado (EVA), cash value added (CVA), flujos de caja descontados, entre otros.

Es importante destacar que dadas las condiciones de la actual economía (economía del conocimiento) se están incluyendo otra serie de indicadores no financieros que permiten identificar si las organizaciones están realmente generando valor con perspectivas futuras. Al hablar de valor con perspectivas futuras, se hace referencia al hecho de que la empresa construya valor con capacidad de generar aún más valor, es decir que le permita sostenerse y crecer en el tiempo, esto siempre con el fin de generar unos beneficios superiores a los esperados por todos los integrantes de una organización (dueños, empleados, clientes y demás).

Edvinsson et al (1998), en el Modelo del Navegador Skandia proponen una serie de indicadores adicionales a los financieros, los cuales denominan índices, que permiten medir la gestión de la empresa en el proceso de creación de valor, pero desde el enfoque del adecuado uso CI; estos índices están relacionados con la satisfacción del cliente, formación de empleados, motivación, liderazgo, cuota del mercado, retención de empleados, eficiencia administrativa, entre otros. 
Kaplan et al (2008) en el Modelo de Balanced Scored Card también proponen un sistema que incluye indicadores no financieros que tienen como objetivo medir la gestión de empresa mediante la generación de valor, estos los agrupa bajo la perspectiva de clientes, procesos internos y aprendizaje. Estas perspectivas guardan una estrecha relación con el capital relacional, capital estructural y capital humano, respectivamente.

En esta investigación se utilizarán indicadores no financieros para medir el valor creado en las empresas del Parque, situación que se soporta teóricamente con los autores mencionados anteriormente.

\section{El capital intelectual y la creación de valor}

Los recursos que afectan el resultado económico de las empresas, pueden clasificarse como activos circulables (corrientes), no circulables (fijos), activos intangibles y CI, cada uno de estos recursos participan en la generación de beneficios de las organizaciones en cierta medida, que sumados darían el total de los beneficios de esta; últimamente se ha hecho indispensable identificar de qué forma los intangibles y el CI generan valor agregado y aumentan la eficiencia en las organizaciones con el fin de tomar decisiones estratégicas. Por lo anterior los activos intangibles y el CI se han convertido en parte importantes del valor de mercado de las empresas y de las organizaciones en general.

Según Ross et al (1997), el valor total de una empresa tiene dos grandes componentes como son: Capital financiero y CI. El capital financiero se refiere a los componentes del valor que se obtienen por metodologías tradicionales de valoración (métodos de los múltiplos, métodos contables, basados en creación de valor, principalmente) y que se puede representar en valores monetarios. El CI se encarga de valorar los componentes intangibles de las organizaciones que generan el soporte de los resultados financieros. La dificultad en relación con este segundo componente radica en la valoración de sus elementos integrantes.

El análisis y estudio especializado de los activos intangibles (CI) surge de los limitantes que tiene la contabilidad tradicional, pues esta no proporciona la información suficiente en relación con su medición y valuación. Con la contabilidad tradicional, la cual solo mide los activos tangibles y reporta resultados de actuación histórica, es difícil lograr la valoración plena de los intangibles y de tal modo no se puede lograr una visión real del potencial de los ingresos para la empresa.

Dado que las medidas financieras no son suficientes para determinar el estado de valor de una empresa, existen otros tipos de medidas que muestran un esquema de valor balanceado entre los recursos financieros e intangibles. "El desarrollo de una nueva arquitectura de medición requiere definir qué tipos de datos necesita medir una empresa acorde con sus estrategias, cómo estos datos pueden generarse y qué reglas deben seguirse para regular su flujo" (Santa y Salas 2014, p. 25). 
Se puede afirmar que en este momento el CI están proporcionando mayor eficiencia y verdaderas fuentes de creación de valor, que suman al valor real que tiene una empresa en el mercado. (Casanueva, Palacios y Val, 2007). "El valor de las futuras ganancias está en los valores intangibles como las personas clave, redes y relaciones, alianzas, cultura y conocimiento" (Edvinsson et al 1998, p.49).

La valoración de los intangibles y del CI busca determinar de la manera más exacta posible el valor de aquellos recursos de la empresa que no son de carácter material y que contribuyen con la generación de riqueza y de valor; mediante la utilización de modelos matemáticos y estadísticos.

Normalmente cuando se utiliza el término valoración de activos en general, se hace referencia al valor económico y financiero de los activos que posee una empresa, es decir a su valor monetario y no en muchas ocasiones a su valor subjetivo; para esta investigación es necesario aclarar que el concepto de valor no se considera un término monetario, sino más bien de percepción entre las relaciones que existen entre las variables a analizar, ya que los activos intangibles son esencialmente activos no financieros que contribuyen de manera significativa con la generación de valor en las organizaciones.

Con el fin de crear valor, los activos intangibles y el CI deben ser trasformados en productos o servicios de los que se derive algún valor. Por lo general, a los activos intangibles se les llama causal de desempeño, lo que sugiere relaciones causales entre estos recursos y la creación de valor organizacional (Meljem et al., 2010, p.82).

Estrada y Dutrénit (2007) citando a Dierickx y Cool (1989) señala que: "En los procesos de creación de valor, los recursos intangibles, tales como la creatividad, el talento o una perspectiva innovadora, pueden generar ventajas competitivas sostenibles dado que son únicos, difíciles de imitar, de naturaleza tácita y complejos" (p.131). Así se asocia el éxito competitivo a la disponibilidad y acumulación de recursos intangibles.

Para medir el impacto del CI en la generación de valor se requiere "transformar la forma como se realiza su medición, pasando de un enfoque tradicional basado en las actividades (medir lo que se hace) a un nuevo enfoque centrado en los resultados (medir lo que se logra y el efecto que se genera)" (Torres, 2005, p. 52). Valorar los activos intangibles y el CI tiene sentido solo en la medida en que se puedan traducir en resultados de negocio como productividad, calidad, servicio al cliente, competitividad, rentabilidad, generación de valor al accionista, entre otros (Torres, 2005, p. 53).

Para Cañibano, García-Ayuso y Sánchez, (1999) el CI se considera generalmente como un determinante fundamental del valor de la empresa y como un elemento asociado estrechamente con la existencia de ventajas competitivas, esto también es apoyado por el Modelo Nova desarrollado por Camisón et al (2003).

Por lo anterior se puede afirmar entonces que una mejora en el CI, entendiéndose que se mejoran sus componentes (capital humano, capital estructural y capital relacional) genera 
impacto positivo en la generación de valor.

\section{Modelo Propuesto}

Como se dijo anteriormente, el CI está conformado por tres componentes: $\mathrm{CH}, \mathrm{CE}$ y CR. Dicha conformación es el resultado no solo de la acumulación diferenciada de cada uno, sino también de la conexión e interrelación entre ellos; es decir que los componentes no son elementos aislados, sino más bien elementos conectados y estrechamente ligados. Sánchez, Melián y Hormiga (2007) citando a Bontis (1998), "certifican la existencia de las mencionadas conexiones" (p.108). Los componentes del capital intelectual no son completamente útiles de manera independiente, su combinación ofrece más ventajas para el beneficio de las empresas. Para Edvinsson et al (1998), el capital intelectual es el resultado de la gestión conjunta del $\mathrm{CH}, \mathrm{CE}$ y CR; La combinación de estos tres componentes es lo que proporciona la fuente de creación de valor para las organizaciones.

Para Sveiby (2007), Bontis, Know y Richardson (2000) y el Modelo de la Universidad West Ontario, existe una causalidad entre los componentes del CI, siendo el componente capital humano la base para el desarrollo de los componentes relacional y estructural; influyendo esto a su vez en los resultados de las organizaciones.

Nonaka (2007); Bueno, Del Real, Fernández, Longo, Merino, Murcia et al (2011) en su modelo Intellectus afirman que la base CI está en el CH y el CR, y estos dan pie al CE; Nonaka (2007) expresa que el conocimiento que tienen los colaboradores $(\mathrm{CH})$ y las relaciones que tiene la empresa (CR) deben conservarse a través de la documentación y normalización de los mismos generando el CE, protegiendo de esa forma que el conocimiento salga de la empresa con la partida de los empleados.

Teniendo en cuenta la fundamentación teórica presentada hasta el momento y de acuerdo a las características y necesidades identificadas en Parquesoft, se necesita un modelo que permita determinar si el CI que actualmente poseen estas empresas, está contribuyendo o no con la generación de valor. Por lo tanto, se propone un modelo que identifique la incidencia que tiene el CI en la generación de valor. Articulando las diferentes piezas del modelo que se han ido presentado a través del documento se tiene:

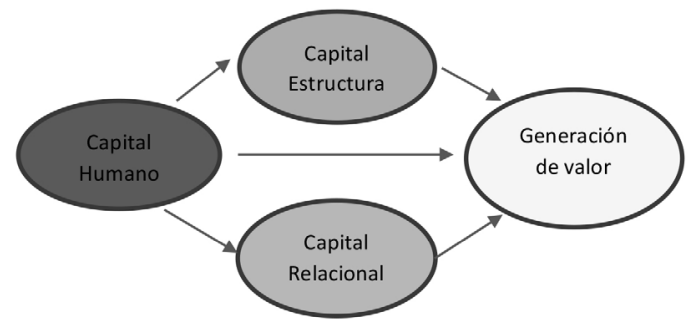

Figura 1. Modelo de generación de valor mediante el CI en las Empresas de Parquesoft Fuente: elaboración propia basado en los conceptos de los autores citados en el apartado 


\section{Metodología de la investigación}

La investigación llevada a cabo es de tipo correlacional, tipo aplicada, es de tipo trasversal vertical, es no experimental y de tipo mixta. Los elementos metodológicos más importantes en el desarrollo de la investigación son:

\section{Construcción de instrumentos de recolección de la información}

Para construir el instrumento de recolección de información, en este caso la encuesta, se determinaron como variables las planteadas en el modelo anterior. Para medir las variables de la investigación, dado que son variables latentes (no observables), se hace uso de varios indicadores observables; para determinar los indicadores a utilizar se realizó una revisión bibliografica, mediante la cual se definieron los siguientes indicadores (ver tablas 1,2,3 y 4):

Tabla 1

Indicadores del capital humano $(\mathrm{CH})$

\begin{tabular}{|c|c|c|c|c|}
\hline Dimensión & Preguntas del cuestionario & $\begin{array}{l}\text { Nombre del } \\
\text { indicador }\end{array}$ & Fuente & Tipo de Escala \\
\hline \multirow{4}{*}{$\begin{array}{l}\text { Trabajo } \\
\text { equipo }\end{array}$} & $\begin{array}{l}\text { Generalmente las ideas de } \\
\text { mejora surgen del trabajo en } \\
\text { equipo }\end{array}$ & CH_TE_V1 & $\begin{array}{l}\text { Construcción Propia } \\
\text { Basado en proyecto } \\
\text { Intelect (1998) }\end{array}$ & Escala de Likert \\
\hline & $\begin{array}{l}\text { Existe un alto grado de con- } \\
\text { fianza entre las personas de } \\
\text { nuestra organización }\end{array}$ & CH_TE_V2 & $\begin{array}{l}\text { Santos et al., } 2011 \\
\text { p. } 83\end{array}$ & Escala de Likert \\
\hline & $\begin{array}{l}\text { Todos en la empresa compar- } \\
\text { timos las misma visión y bus- } \\
\text { camos alcanzar los mismos } \\
\text { objetivos }\end{array}$ & CH_TE_V3 & $\begin{array}{l}\text { Construcción propia, } \\
\text { basado Delgado et al } \\
\text { (2011) p. } 219\end{array}$ & Escala de Likert \\
\hline & $\begin{array}{l}\text { Nuestros colaboradores se } \\
\text { apoyan para formular nuevas } \\
\text { ideas, solucionar problemas o } \\
\text { incrementar su capacidad de } \\
\text { trabajo diario. }\end{array}$ & CH_TE_V4 & $\begin{array}{l}\text { Construcción propia, } \\
\text { basado Delgado et al } \\
\text { (2011) p. } 219\end{array}$ & Escala de Likert \\
\hline
\end{tabular}




\begin{tabular}{|c|c|c|c|c|}
\hline \multirow{5}{*}{$\begin{array}{l}\text { Formación y } \\
\text { capacitación }\end{array}$} & Ingreso por colaborador & CH_FyC_V3 & Roos et al (1997) & Escala de razón \\
\hline & $\begin{array}{l}\text { Horas de formación por em- } \\
\text { pleado }\end{array}$ & CH-FyC_V1 & Roos et al (1997) & Escala de razón \\
\hline & $\begin{array}{l}\text { Gastos en formación por em- } \\
\text { pleado }\end{array}$ & CH_FyC_V2 & Roos et al (1997) & Escala de razón \\
\hline & $\begin{array}{l}\text { Nuestro colaboradores tienen } \\
\text { un alto dominio del idioma } \\
\text { ingles }\end{array}$ & CH_FyC_V5 & Propio & Escala de Likert \\
\hline & $\begin{array}{l}\text { Nuestros colaboradores po- } \\
\text { seen un alto grado de compe- } \\
\text { tencia en técnicas informáti- } \\
\text { cas y tecnológicas }\end{array}$ & CH_FyC_V4 & $\begin{array}{l}\text { Martín et al (2009) } \\
\text { p. } 108\end{array}$ & Escala de Likert \\
\hline \multirow{3}{*}{$\begin{array}{l}\text { Cultura de la } \\
\text { Innovación }\end{array}$} & $\begin{array}{l}\text { Veo nuestra empresa como } \\
\text { innovadora, con voluntad de } \\
\text { nuevos experimentos y coraje } \\
\text { para correr riesgos. }\end{array}$ & CH_CIn_V1 & Santos (2011) p. 80 & Escala de Likert \\
\hline & $\begin{array}{l}\text { Conseguimos extraer valor } \\
\text { del proceso de innovación }\end{array}$ & CH_CIn_V2 & $\begin{array}{l}\text { Santos et al (2011) } \\
\text { p. } 80\end{array}$ & Escala de Likert \\
\hline & $\begin{array}{l}\text { Nuestra empresa tiene un am- } \\
\text { biente de trabajo que propicia } \\
\text { la participación activa de las } \\
\text { personas en la innovación de } \\
\text { la empresa. }\end{array}$ & CH_CIn-V3 & $\begin{array}{l}\text { Santos et al (2011) } \\
\text { p. } 80\end{array}$ & Escala de Likert \\
\hline \multirow{2}{*}{$\begin{array}{l}\text { Sistemas de } \\
\text { remuneración } \\
\text { e incentivos }\end{array}$} & $\begin{array}{l}\text { Nuestra empresa otorga in- } \\
\text { centivos a los empleados in- } \\
\text { novadores o que aportan ideas }\end{array}$ & CH_RI_V1 & $\begin{array}{l}\text { Martín et al (2009) } \\
\text { p.108 }\end{array}$ & Escala de Likert \\
\hline & $\begin{array}{l}\text { Nuestros colaboradores tienen } \\
\text { un alto grado de satisfacción }\end{array}$ & CH-RI_V2 & $\begin{array}{l}\text { Martín et al (2009) } \\
\text { p. } 96\end{array}$ & Escala de Likert \\
\hline
\end{tabular}

Fuente: elaboración propia tomando los conceptos de los autores citados 
Tabla 2

Indicadores del capital estructural (CE)

\begin{tabular}{|c|c|c|c|c|}
\hline Dimensión & Preguntas del cuestionario & $\begin{array}{l}\text { Nombre del } \\
\text { Indicador }\end{array}$ & Fuente & Tipo de Escala \\
\hline \multirow{4}{*}{$\begin{array}{l}\text { Tecnologías de } \\
\text { la Información } \\
\text { y comunica- } \\
\text { ción }\end{array}$} & Manejamos buenas prácticas & CE_TIC_V1 & Construcción Propia & Escala de Likert \\
\hline & $\begin{array}{l}\text { Nuestra organización usa re- } \\
\text { gistro y licencias como ma- } \\
\text { nera de conservar y proteger } \\
\text { el conocimiento }\end{array}$ & CE_TIC_V2 & Construcción Propia & Escala de Likert \\
\hline & $\begin{array}{l}\text { Nuestros equipos y aplicacio- } \\
\text { nes son de última tecnología }\end{array}$ & CE_TIC_V3 & Construcción Propia & Escala de Likert \\
\hline & $\begin{array}{l}\text { Nuestra organización cuenta } \\
\text { con canales óptimos de co- } \\
\text { municación interna }\end{array}$ & CE_TIC_V4 & Construcción Propia & Escala de Likert \\
\hline Dimensión & Preguntas del cuestionario & $\begin{array}{c}\text { Nombre del } \\
\text { Indicador }\end{array}$ & Fuente & Tipo de Escala \\
\hline \multirow{3}{*}{$\begin{array}{l}\text { Investigación y } \\
\text { Desarrollo }\end{array}$} & $\begin{array}{l}\text { El número de desarrollos e } \\
\text { innovaciones tecnológicas ha } \\
\text { sido alto en los últimos tres } \\
\text { años }\end{array}$ & CE_ID_V1 & $\begin{array}{l}\text { Adaptado de Alama et } \\
\text { al (2009) p. } 10\end{array}$ & Escala de Likert \\
\hline & $\begin{array}{l}\text { Nuestra organización tiene } \\
\text { conocimiento para competir } \\
\text { en un mercado global }\end{array}$ & CE_ID_V2 & $\begin{array}{l}\text { Alama et al (2009) p. } \\
12\end{array}$ & Escala de Likert \\
\hline & $\begin{array}{l}\text { Nuestra empresa tiene un } \\
\text { conjunto de procesos y pro- } \\
\text { cedimientos centrados en } \\
\text { impulsar el aprendizaje y la } \\
\text { innovación }\end{array}$ & CE_ID_V3 & $\begin{array}{l}\text { Santos et al. (2011) } \\
\text { p. } 80\end{array}$ & Escala de Likert \\
\hline \multirow{2}{*}{$\begin{array}{l}\text { Estructura or- } \\
\text { ganizacional } \\
\text { flexible }\end{array}$} & $\begin{array}{l}\text { Nuestros colaboradores tie- } \\
\text { nen la capacidad de desarro- } \\
\text { llar diversas funciones en la } \\
\text { empresa }\end{array}$ & CE_EOF_V1 & Construcción propia & Escala de Likert \\
\hline & $\begin{array}{l}\text { Tenemos descripciones de- } \\
\text { talladas de las tareas, proce- } \\
\text { dimientos y de las políticas } \\
\text { para guiar la acción de los } \\
\text { colaboradores }\end{array}$ & CE_EOF_V2 & $\begin{array}{l}\text { Santos et al (2011) p. } \\
80\end{array}$ & Escala de Likert \\
\hline
\end{tabular}

Fuente: elaboración propia tomando los conceptos de los autores citados 
Tabla 3

Indicadores del capital relacional (CR)

\begin{tabular}{|c|c|c|c|c|}
\hline Dimensión & Preguntas del cuestionario & $\begin{array}{l}\text { Nombre del } \\
\text { indicador }\end{array}$ & Fuente & Tipo de Escala \\
\hline \multirow{4}{*}{$\begin{array}{l}\text { Participación en } \\
\text { redes }\end{array}$} & $\begin{array}{l}\text { La participación en redes ha } \\
\text { permitido a la organización } \\
\text { desarrollar soluciones y mejo- } \\
\text { ras en los procesos y productos } \\
\text { en los últimos años. }\end{array}$ & CR_PR-V1 & $\begin{array}{l}\text { Construcción } \\
\text { propia }\end{array}$ & Escala de Likert \\
\hline & $\begin{array}{l}\text { Nuestra empresa tiene habili- } \\
\text { dades para establecer alianzas }\end{array}$ & CR_PR-V2 & $\begin{array}{l}\text { Martín et al } \\
\text { (2009) p. } 109\end{array}$ & Escala de Likert \\
\hline & $\begin{array}{l}\text { Número de acuerdos activos y } \\
\text { solidos de colaboración }\end{array}$ & CR_PR_V3 & $\begin{array}{l}\text { Naranjo et al } \\
\text { (2013) p. } 48\end{array}$ & Métrica \\
\hline & $\begin{array}{l}\text { Prevalece la búsqueda de rela- } \\
\text { ciones a largo plazo }\end{array}$ & CR_PR_V4 & $\begin{array}{l}\text { Román et al } \\
\text { (2013) p. } 361\end{array}$ & Escala de Likert \\
\hline \multirow{4}{*}{$\begin{array}{l}\text { Relación } \\
\text { clientes }\end{array}$} & $\begin{array}{l}\text { Nuestra empresa posee una } \\
\text { cartera amplia de clientes fre- } \\
\text { cuentes }\end{array}$ & CE_RC_V1 & $\begin{array}{l}\text { Martín et al } \\
\text { (2009) p. } 100\end{array}$ & Escala de Likert \\
\hline & $\begin{array}{l}\text { Por lo general las relaciones } \\
\text { con clientes son a largo plazo }\end{array}$ & CE_RC_V2 & $\begin{array}{l}\text { Martín et al } \\
\text { (2009) p. } 100\end{array}$ & Escala de Likert \\
\hline & $\begin{array}{l}\text { Trabajamos conjuntamente } \\
\text { con nuestros clientes para de- } \\
\text { sarrollar soluciones }\end{array}$ & CE_RC_V3 & $\begin{array}{l}\text { Delgado et al } \\
(2011) \text { p. } 219\end{array}$ & Escala de Likert \\
\hline & $\begin{array}{l}\text { Porcentaje de las ideas aporta- } \\
\text { das por clientes }\end{array}$ & CE_RC_V4 & $\begin{array}{l}\text { Construcción } \\
\text { propia }\end{array}$ & Porcentaje \\
\hline \multirow{3}{*}{$\begin{array}{l}\text { Relación } \\
\text { proveedores }\end{array}$} & $\begin{array}{l}\text { Las relaciones de nuestra em- } \\
\text { presa con proveedores son ge- } \\
\text { neralmente a largo plazo }\end{array}$ & CE_RP_V1 & $\begin{array}{l}\text { Martín et al } \\
\text { (2009) p } 109\end{array}$ & Escala de Likert \\
\hline & $\begin{array}{l}\text { Trabajamos de manera con- } \\
\text { junta con nuestros proveedo- } \\
\text { res para solucionar problemas }\end{array}$ & CE_RP_V2 & $\begin{array}{l}\text { Martín et al } \\
\text { (2009) p. } 109\end{array}$ & Escala de Likert \\
\hline & $\begin{array}{l}\text { Ideas aportadas por provee- } \\
\text { dores }\end{array}$ & CE_RP_V3 & $\begin{array}{l}\text { Naranjo et al } \\
(2013) \text { p. } 48\end{array}$ & Métrica \\
\hline
\end{tabular}

Fuente: elaboración propia tomando los conceptos de los autores citados 
Tabla 4

Indicadores de generación de valor (GV)

\begin{tabular}{|c|c|c|c|c|}
\hline Dimensión & Preguntas del cuestionario & $\begin{array}{l}\text { Nombre del } \\
\text { indicador }\end{array}$ & Fuente & Tipo de Escala \\
\hline \multirow[b]{2}{*}{ Ventas } & Su empresa exportó en el año 2015 & GV-V1 & Elaboración propia & Pregunta dicótoma \\
\hline & $\begin{array}{l}\text { El ingreso por ventas por colabora- } \\
\text { dor en el año } 2015 \text { (Total de ventas } \\
\text { \$/total colaboradores }\end{array}$ & GV-V2 & Elaboración propia & Pregunta ordinal \\
\hline \multirow{5}{*}{ Innovación } & $\begin{array}{l}\text { Cantidad de nuevos productos de- } \\
\text { sarrollados entre los años } 2013 \text { y } \\
2015\end{array}$ & GV-V3 & $\begin{array}{l}\text { OCDE (2005). pp. } \\
34-37\end{array}$ & Escala de razón \\
\hline & $\begin{array}{l}\text { Cantidad de nuevos servicios de- } \\
\text { sarrollados entre los años } 2013 \text { y } \\
2015\end{array}$ & GV_V4 & $\begin{array}{l}\text { OCDE (2005). pp. } \\
34-37\end{array}$ & Escala de razón \\
\hline & $\begin{array}{l}\text { Cantidad mejoras significativas de } \\
\text { productos desarrollados entre los } \\
\text { años } 2013 \text { y } 2015\end{array}$ & GV_V5 & $\begin{array}{l}\text { OCDE (2005). pp. } \\
34-37\end{array}$ & Escala de razón \\
\hline & $\begin{array}{l}\text { Cantidad de mejoras significativas } \\
\text { de Servicios desarrollados entre } \\
\text { los años } 2013 \text { y } 2015\end{array}$ & GV_V6 & $\begin{array}{l}\text { OCDE (2005). pp. } \\
34-37\end{array}$ & Escala de razón \\
\hline & $\begin{array}{l}\text { Cantidad de nuevos canales de co- } \\
\text { mercialización desarrollados entre } \\
\text { los años } 2013 \text { y } 2015\end{array}$ & GV_V7 & $\begin{array}{l}\text { OCDE (2005). pp. } \\
34-37\end{array}$ & Escala de razón \\
\hline $\begin{array}{l}\text { Colabora- } \\
\text { dores }\end{array}$ & $\begin{array}{l}\text { El número de colaboradores entre } \\
\text { los años } 2013 \text { y } 2015\end{array}$ & $\mathrm{GV}_{-} \mathrm{V8}$ & Elaboración propia & Escala ordinal \\
\hline
\end{tabular}

Fuente: elaboración propia tomando los conceptos de los autores citados

\section{Población y muestra}

Como se mencionó anteriormente las empresas objeto de estudio son las Empresas de Parquesoft Colombia, la ficha técnica de la muestra se presenta en la tabla 5.

Tabla 5

Ficha técnica de la muestra

\begin{tabular}{ll}
\hline Fecha de aplicación de la encuesta & Febrero 2 a abril 14 de 2016 \\
\hline Población & 140 empresas \\
\hline Tamaño de la muestra & 80 instrumentos validos \\
\hline Herramienta de recolección de información & Encuesta no continua y activa \\
\hline
\end{tabular}




\begin{tabular}{ll}
\hline Procedimiento de muestreo & $\begin{array}{l}\text { Se envió el cuestionario a todas las empresas de la pobla- } \\
\text { ción, a través de un link de Google Formularios }\end{array}$ \\
\hline Medio de aplicación de la encuesta & Correo electrónico (envió de link) y contacto telefónico \\
\hline Encuesta diligenciada por: & Representante legal de la empresa \\
\hline $\begin{array}{l}\text { Software utilizados para recolectar y analizar } \\
\text { la información }\end{array}$ & Formularios Google \\
& Smart-PLS 2.0 \\
\hline
\end{tabular}

Fuente elaboración propia

\section{Metodología para el análisis de la información}

Al revisar la literatura existente sobre los modelos teóricos que analizan el CI, se evidencia que cada uno presenta estructuras diferentes, ofrecen resultados diferentes (resultados monetarios, indicadores, de valor), utilizan diferentes variables de análisis y que básicamente dependen de las condiciones específicas de la empresa que se va a valorar; por lo tanto, se puede concluir que no existe un método único de valoración o análisis del CI.

De igual forma, se identificó con la revisión literaria la existencia de una serie de autores que utilizan otras herramientas metodológicas para identificar las relaciones entre los componentes del CI y las variables que se desean estudiar. Las herramientas identificadas, son las técnicas de análisis estadístico multivalente (técnicas estadísticas de segunda generación), entre ellas Modelos de Ecuaciones Estructurales (MEE). Barletta, Pereira, Yoguel y Robert (2013); Ospina, Berrio, Bedoya, Cárdenas y Muñoz (2013); Motta, Zavaleta, Llinás, y Luque (2013).

Para analizar los datos de la investigación y validar el modelo propuesto se hará uso de los MEE. Los MEE son técnicas multivariantes que combinan aspectos de la regresión múltiple (examinando relaciones de dependencia) y análisis factorial (que representan conceptos inmedibles -factores- con variables múltiples) para estimar una serie de relaciones de dependencia interrelacionadas simultáneamente." Cepeda, G. (2004). Pp.4

Los MEE pueden analizarse a través de dos técnicas estadísticas, para esta investigación se optó utilizar la técnica de análisis basados en componentes o Partial Least Squares (PLS), por considerarse la apropiada de acuerdo a las características de las variables de la investigación. Para el análisis se utilizó el software Smart-PLS 2.0.

\section{Evaluación de los resultados de la investigación}

Los datos analizados provienen de las encuestas realizadas a las empresas, estos se organizaron y tabularon de acuerdo a las exigencias del software SmartPLS 2.0 para ser analizados. 
Para validar el modelo a través de la técnica PLS de MEE, es necesario en una primera etapa realizar una evaluación de los modelos de medida (indicadores), que consiste en determinar si los conceptos teóricos están medidos correctamente a través de las variables observadas, esta evaluación tiene el fin de demostrar la solidez del instrumento (encuesta) de medición utilizado.

En la segunda etapa se hace la evaluación del modelo estructural, esto consiste en determinar de qué forma la información empírica obtenida con la investigación soporta los conceptos teóricos, y de esta forma definir si la teoría ha sido empíricamente confirmada; esto es examinar las capacidades predictivas del modelo y la relación entre sus constructos.

En la tabla 6 se presentan las evaluaciones que se hacen en cada una de las etapas y los resultados esperados.

Tabla 6

Análisis aplicados en el MEE

\begin{tabular}{|c|c|c|}
\hline Etapa & Procedimiento & Valores validos \\
\hline \multirow{5}{*}{$\begin{array}{l}\text { Etapa 1: Valora- } \\
\text { ción de la validez } \\
\text { y fiabilidad del } \\
\text { modelo }\end{array}$} & Firhilided de consistencis interno & \\
\hline & Fraomada de consistencra miena & $\cdot$ Fiabilidad compuesta $(\varrho c)>0.7$ \\
\hline & Fiabilidad individual del ítem & Cargas $\lambda>=0.5$ \\
\hline & Validez convergente & Varianza media extraída (AVE > 0.5). \\
\hline & Validez Discriminante & Criterio de Cargas cruzadas. \\
\hline \multirow{4}{*}{$\begin{array}{l}\text { Etapa 2: Valida- } \\
\text { ción del modelo } \\
\text { estructural }\end{array}$} & Coeficiente de Determinación $\mathrm{R}^{2}$ & $\begin{array}{l}\text { Identifica valores de } 0.67,0.33 \text { y } 0.19 \text {, denotados } \\
\text { como sustanciales, moderados y débiles. }\end{array}$ \\
\hline & Coeficientes Path $\beta$ & $\begin{array}{l}\text { Los coeficientes path que alcanzan al menos un } \\
\text { valor de } 0.2 \text { y son ideales si son mayores a } 0.3\end{array}$ \\
\hline & Análisis Bootstraping & $\begin{array}{l}\text { Permite al análisis "t" de Student ser ejecutado } \\
\text { para la significancia de las relaciones del modelo } \\
\text { path. Son significativos los indicadores cuyo "t" } \\
\text { de Student es mayor que } 1,96\end{array}$ \\
\hline & Valor $Q^{2}$ & $\begin{array}{l}\text { Valores de } 0.02,0.15 \text {, y } 0.35 \text { revelan pequeña, } \\
\text { media o gran relevancia predictiva }\end{array}$ \\
\hline
\end{tabular}

Fuente: elaboración propia tomando los conceptos de Cepeda y Roldán, (2004) 
A continuación, se presenta el nomograma del modelo:

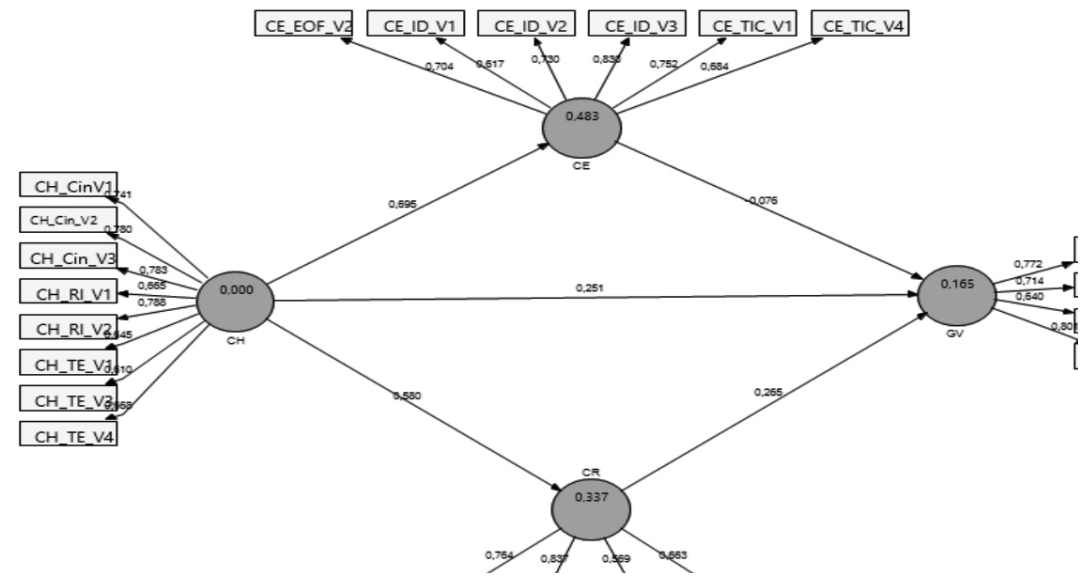

Figura 2. Nomograma con cargas individuales

Fuente: elaboración propia utilizando SMART-PLS 2.0. Ringle, Wende y Will (2005)

\section{Valoración de la validez y fiabilidad del modelo}

- Fiabilidad de consistencia interna: Como se ve en la tabla 7 la fiabilidad compuesta de cada uno de los constructos dio un valor superior a 0.7, esto muestra que los indicadores que componen cada uno de dichos factores están relacionados entre sí. En la tabla 7 los constructos $\mathrm{CE}, \mathrm{CH}$ y GV dieron valores del Alpha de Cronbach superiores al valor mínimo aceptado, significando que los indicadores de cada uno de ellos están relacionados con su respectivo constructo y dándole validez a cada uno de ellos. La variable CR arrojó un valor de 0.68 , menor al mínimo esperado, para efectos de la investigación y dado que en el análisis de fiabilidad interna obtuvo un valor aceptable no se elimina de la investigación y con esto también se garantiza preservar la validez de contenido.

- Validez convergente: En la tabla 7 todos los constructos presentan un valor AVE mayor que 0,5 , esto quiere decir que los diferentes ítems destinados a medir un concepto o constructo miden realmente lo mismo, entonces el ajuste de dichos ítems es significativo y están altamente correlacionados. 
Tabla 7

Análisis de fiabilidad y validez

\begin{tabular}{|c|c|c|c|}
\hline \multirow{2}{*}{ Variable o constructo } & \multirow{2}{*}{ Validez convergente (AVE) } & \multicolumn{2}{|c|}{ Fiabilidad de consistencia Interna } \\
\hline & & Fiabilidad compuesta & Alpha de cronbach \\
\hline $\mathrm{CE}$ & 0.5218 & 0.8666 & 0.81 \\
\hline $\mathrm{CH}$ & 0.5088 & 0.8915 & 0.86 \\
\hline $\mathrm{CR}$ & 0.5119 & 0.8043 & 0.68 \\
\hline GV & 0.5390 & 0.8228 & 0.73 \\
\hline
\end{tabular}

Fuente: elaboración propia utilizando SMART-PLS 2.0. Ringle, Wende y Will (2005)

- Fiabilidad individual del ítem: en la figura 2, los valores que están en las flechas que apuntan a los indicadores (cuadros) son las cargas $\lambda$, las cuales dan mayores a 0.5. Los indicadores de la figura 2 son menos que los indicadores presentados en la tablas $1,2,3$ y 4, esto se debe a que se hizo una depuración de indicadores, eliminando aquellos que tuvieran una carga $\lambda$ menor que 0.5 . Una carga mayor que 0.5 significa que dicho ítem contribuye de manera significativa en la construcción de la variable latente.

- Validez discriminante: analizando el resultado de cargas cruzadas (ver tabla 8 y 9) se obtuvo que cada indicador presenta una correlación más alta con su propia variable latente antes que con otras, esto indica en qué medida un constructo dado es diferente de otros constructos. La raíz cuadrada de AVE de cada constructo es mayor que la correlación entre ellos, por lo tanto, se puede concluir que el modelo cumple con el criterio de validez discriminante y que las variables latentes están diferenciadas con claridad.

Tabla 8

Raíz cuadrada de AVE

\begin{tabular}{ll}
\hline Constructo & Raíz cuadrada AVE \\
\hline CE & 0.7224 \\
CH & 0.7133 \\
CR & 0.7155 \\
GV & 0.7342 \\
\hline
\end{tabular}

Fuente: elaboración propia utilizando SMART-PLS 2.0. Ringle, Wende y Will (2005) 
Tabla 9

Correlaciones entre constructos. Criterio de Fornell-Larcker

\begin{tabular}{lllll}
\hline & $\mathrm{CE}$ & $\mathrm{CH}$ & $\mathrm{CR}$ & $\mathrm{GV}$ \\
\hline $\mathrm{CE}$ & 0.7224 & & & \\
$\mathrm{CH}$ & 0.6953 & 0.7133 & & \\
$\mathrm{CR}$ & 0.6055 & 0.5802 & 0.7155 & \\
$\mathrm{GV}$ & 0.2587 & 0.3517 & 0.3646 & 0.7342 \\
\hline
\end{tabular}

Fuente: elaboración propia utilizando SMART-PLS 2.0. Ringle, Wende y Will (2005)

Partiendo de los resultados mostrados en este apartado, se puede concluir que las escalas analizadas para medir las dimensiones del capital humano, capital estructural, capital relacional y la generación de valor son válidas y fiables.

\section{Validación del modelo estructural}

- Coeficiente $\mathrm{R}^{2}$ : Los valores que aparecen dentro de los círculos (variables latentes endógenas) (figura 2) son el valor $\mathrm{R}^{2}$, como se puede ver presentan valores mayores 0.1 , esto indica que el modelo tiene capacidad predictiva sobre las variables endógenas del mismo.

- Coeficientes path del modelo: son los valores que están en las flechas que unen las variables latentes (círculos figura 2), los valores que sean inferiores a 0.2 significan que la variable que la variable exógena no tiene un impacto significativo sobre la variable endógena.

- Análisis Bootstraping arrojo los siguientes resultados ver tabla 9.

Tabla 9

Analisis Bootstraping

\begin{tabular}{|c|c|c|c|}
\hline Relación entre constructos & Error estándar & T-Student & Nivel de significancia \\
\hline $\mathrm{CE}->\mathrm{GV}$ & 0.1958 & 0.3906 & No significativa \\
\hline $\mathrm{CH}->\mathrm{CE}$ & 0.0547 & 12.7005 & Significativa \\
\hline $\mathrm{CH}->\mathrm{CR}$ & 0.0712 & 8.1552 & Significativa \\
\hline $\mathrm{CH}->\mathrm{GV}$ & 0.1248 & 2.0113 & Significativa \\
\hline $\mathrm{CR}->\mathrm{GV}$ & 0.1285 & 2.0647 & Significativa \\
\hline
\end{tabular}

Fuente: elaboración propia utilizando SMART-PLS 2.0. Ringle, Wende y Will (2005) 
Como se ve en la tabla anterior, el análisis bootstrapping para el modelo, confirma el resultado dado por los coeficients path $\beta$, donde la relación entre el constructo Capital estructural y la generación de valor no es significativa.

Valor $\mathrm{Q}^{2}$ : este valor mide la capacidad predictiva del modelo, la tabla 9 muestra que los valores $\mathrm{Q}^{2}$ de los constructos endógenos son superiores a cero, indicando la capacidad de predicción del modelo.

Tabla 10

Valor $\mathrm{Q}^{2}$

\begin{tabular}{llll}
\hline Total & SSO & SSE & $\mathrm{Q}^{2}=1-\mathrm{SSE} / \mathrm{SSO}$ \\
\hline $\mathrm{CE}$ & 480 & 363.12 & 0.2435 \\
$\mathrm{CH}$ & 640 & 640.00 & \\
$\mathrm{CR}$ & 320 & 268.59 & 0.1606 \\
$\mathrm{GV}$ & 320 & 293.75 & 0.0820 \\
\hline
\end{tabular}

Fuente: elaboración propia utilizando SMART-PLS 2.0. Ringle, Wende y Will (2005)

Teniendo en cuenta lo presentado en este apartado se puede afirmar que la información empírica obtenida con la investigación soporta los conceptos teóricos, el modelo tiene capacidad predictiva y existe relación entre sus constructos; es decir tienen significancia estadística.

\section{Análisis cualitativo de los resultados}

En el apartado anterior se presentan los resultados de la evaluación del modelo, en este numeral se hará un análisis interpretativo de tipo cualitativo de cada uno de dichos resultados en aras de validar el modelo propuesto.

Capital Humano: Para medir el capital humano, dado que es una variable latente exógena, se propuso inicialmente medirlo con 14 indicadores distribuidos en cuatro dimensiones (ver tabla 1), pero después de evaluar los modelos de medida en donde se demuestra la solidez del instrumento de medición utilizado, se eliminaron 6 indicadores. Es importante destacar que todos los indicadores relacionados con la dimensión formación y capacitación se eliminaron, situación que genera luces de alarma en cuanto a la fortaleza del CH, ya que la formación y conocimiento de los colaboradores son elementos fundamentales en dicho constructo. Esto permite inferir ya sea que las empresas no están destinados recursos y esfuerzos en ello, o que lo que se destina para ello no está generando el impacto necesario.

Los indicadores que más le aportan a la construcción del indicador son los relacionados con la dimensión cultura de la innovación, pues presentan las cargas más altas, todas superiores a 0.7 (ver grafica 2). Esto reafirma la naturaleza de las empresas del Parque, empresas en función de la innovación en sus productos, procesos y servicios. 
En segundo orden de importancia en cuanto a la aportación de la construcción del CH, se tienen la dimensión remuneración e incentivos, destacándose el grado de satisfacción por parte de los colaboradores en la empresa.

En cuanto al tercer y último componente, trabajo en equipo, todos los indicadores presentan cargas superiores a 0.6 , siendo valores aceptables, pero no los más óptimos; esto conlleva a pensar que se debe fomentar y mejorar los espacios de trabajo en equipo pues es allí donde se crea y transfiere el conocimiento y se dan los procesos de innovación.

Capital Estructural: El capital estructural es una variable latente endógena, que depende del constructo Capital humano y a su vez alimenta al constructo generación de valor, tal y como se definió en el modelo.

Para medir el capital estructural se plantearon inicialmente 9 indicadores, después de realizar el análisis de fiabilidad individual de cada ítem, se eliminaron 3 indicadores, dos pertenecientes a la dimensión tecnologías de la información y comunicación (TIC) y uno a la dimensión estructura organizacional flexible.

La dimensión que más contribuye con este constructo es “investigación y desarrollo", lo que ratifica la razón de ser de las empresas del Parque, destacándose principalmente la existencia de espacios, procesos y procedimientos para investigar y desarrollar ideas; esta dimensión se refuerza y se ratifica con la dimensión "cultura de la innovación” del constructo Capital humano, en donde también ocupa un papel importante dentro de su constructo.

Este constructo, tal y como se mostró mediante el análisis MEE presentado, no contribuye a explicar la generación de valor al interior de las empresas del parque.

Capital relacional: Es el componente del CI que más contribuye y explica el constructo generación de valor; para este constructo se plantearon inicialmente 12 indicadores, de los cuales finalmente se dejaron en el modelo 4 (indicadores).

La dimensión que más se destaca en este caso es la de "participación en redes", pues presenta las cargas más altas en cuanto a su aporte al constructo, confirmando que el número de vínculos que tienen las empresas se relaciona positivamente con el nivel de generación de valor; esto aumenta las posibilidades de abrir nuevos mercados y mejorar el desempeño competitivo de las empresas.

La dimensión "relación con clientes", a pesar de que contribuye, se hace de manera débil, situación que se corrobora con el hecho de que las empresas tienen mayormente modelos de negocio enfocados al desarrollo de productos y servicios propios.

Generación de valor Para este constructo se propusieron inicialmente 8 indicadores, quedando finalmente cuatro indicadores de las dimensiones innovación y colaboradores; es importante destacar que estos resultados validan una de las características fundamentales de las EBT de software, que es precisamente la innovación. 


\section{Conclusiones}

La investigación se centró en el estudio del CI, el cual como se argumentó, está compuesto por tres elementos altamente interrelacionados que son el $\mathrm{CH}$, el $\mathrm{CE}$ y el $\mathrm{CR}$; siendo el $\mathrm{CH}$ la base que soporta los demás capitales y por la tanto es la base del CI; adicionalmente se evidencio que CI juega un papel fundamental en la actual economía del conocimiento, al ser un activo que contribuye de manera significativa en los procesos de generación de valor al interior de las organizaciones.

Los MEE permitieron identificar las relaciones causales entre los componentes del capital intelectual de las empresas objeto de estudio y la generación de valor al interior de ellas, pues facilitó la articulación de teoría y de los datos empíricos obtenidos.

El modelo propuesto plantea que los componentes del CI, en este caso el CH, el CE y el CR contribuyen positivamente con la generación de valor al interior de las empresas de base tecnológica de software; este modelo validó empíricamente, pues se mostró a través del análisis estadístico multivariado realizado que el 8,83\% del constructo generación de valor es explicado y construido por el capital humano y el 9,66\% del mismo, por el capital relacional; por el contrario el capital estructural no afecta de manera positiva con la generación de valor.

Se considera que uno de los principales aportes de este trabajo fue estructurar de manera coherente el capital CI para las empresas objeto de estudio, mediante la identificación de sus componentes y la relación entre ellos, proponiendo una serie de indicadores que permitieron realizar procesos de medición de los mismos. Otro aporte importante fue determinar las relaciones causales de los componentes del CI con la generación de valor.

Como conclusión general se tiene que el modelo propuesto comprueba la teoría y tiene la capacidad predictiva del comportamiento de las constructos endógenos de la investigación, esto permitió determinar que los componentes del CH y CR, del CI, contribuyen positivamente con la generación de valor, aunque es importante resaltar que solo explican el $18.49 \%$ del valor que se genera en las empresas del parque; este resultado permite concluir que existen otra serie de elementos que también contribuyen con la GV y no se tuvieron en cuenta en la investigación. Esta investigación permitió contrastar el papel estratégico que juega el CI en la obtención de valor en las empresas. Este modelo permite a las empresas del Parque darse cuenta de que forma los esfuerzos que se realizan en el fortalecimiento del CI impactan en la generación de valor. 


\section{Referencias}

Alama, E. M. (2009). Capital intelectual y resultados empresariales en las empresas de servicios profesionales de España. Tesis de la Universidad Complutense de Madrid, Facultad de Ciencias Económicas y Empresariales, Departamento de Organización de Empresas.

Álvarez, C. (2010). Hacia un nuevo modelo de valoración de intangibles. Trabajo de grado Doctorado, Ciencias de la Comunicación, Universitat Jaume. Castellón.

Barletta, F., Pereira, M., Yoguel, G., y Robert, V. (2013). Argentina: Dinámica reciente del sector de software y servicios informáticos. Revista CEPAL. https://doi.org/10.18356/2813fe44-es

Bontis, N., Know, W., y Richardson, S. (2000). Intellectual capital and business performance in Malaysian industries. Journal of Intelectual Capital, 1(1), pp 85-100. https://doi.org/10.1108/14691930010324188

Brooking, A. (1996). Intellectual Capital: Core Asset for the Third Millennium Enterprise. London: International Thomson Business Press. https://doi.org/10.1016/S0024-6301(97)80917-X

Bueno, E., Del Real, H., Fernández, P., Longo, M., Merino, C., Murcia, C. y Salmador, M. (2011). Modelo Intellectus de Medición, gestión e información del capital intelectual. (Nueva versión actualizada). Universidad Autónoma de Madrid.

Camisón, Z. C., Lapiedra, A. R., Segarra, M. y Boronat, N. M. (2003). Marco conceptual de la relación entre innovación y tamaño organizativo. Revista Madrid, No.9, octubre-noviembre. http://www.madrimasd.org/revista/ revista19/tribuna/tribuna1 .asp.

Cañibano, L. García-Ayuso, y M. Sánchez, P. (1999). La relevancia de los intangibles para la valoración y la gestión de empresas: revisión de la literatura (1). Revista Española de Financiación y Contabilidad, 100, 17-88.

Casanueva, C., Palacios, M. y Val, T. (2007). Inversión en Nuevas empresas de base Tecnológica en la comunidad de Madrid, [en línea]. Madrid. Recuperado 9 de septiembre de 2011, de www.ingor.etssi.upm.es/administración,html

Cepeda, G.; Roldán, J.L. (2004). "Aplicando en la Práctica la Técnica PLS en la Administración de Empresas", Congreso de la ACEDE, septiembre 19, 20 y 21, Murcia, España.

Colciencias (2008). Las empresas de base tecnológica e innovadoras y su relación con los fondos de inversión en capital. Convenio BID-Colciencias-Programa Finbatec. Bogotá: Javegraf.

Delgado, M., Martín de Castro, G., Navas, J. E., y Cruz, J. (2011). Capital social, capital relacional e innovación tecnológica. Una aplicación al sector manufacturero español de alta y media-alta tecnología. Cuadernos de Economía y Dirección de la Empresa, 14(4), 207-221. https://doi.org/10.1016/j.cede.2011.04.001

Edvinsson, L. y Malone, M. S. (1998). El capital intelectual: Cómo identificar y calcular el valor de los recursos intangibles de su empresa. Colombia: Editorial Norma S.A.

Estrada, S. y Dutrénit, G. (2007). Gestión del conocimiento en Pymes y desempeño competitivo. Engevista, 9(2), pp. 129-148. https://doi.org/10.22409/engevista.v9i2.203

Kaplan, R. y Norton, D. (2008). Mapas Estratégicos: convirtiendo activos intangibles, en resultados tangibles. Barcelona: Ediciones Gestión 2000.

Lanzas, A. (2016). Modelo para medir el impacto del capital humano en los resultados de la innovación en un grupo de empresas de base tecnológica de software, Ponencia, XXI Congreso Internacional de Contaduría, Administración e Informática, Universidad Nacional Autónoma de México.

Martín de Castro, G; Alama, E; Navas, J; López, P. (2009). El papel del capital intelectual en la innovación tecnológica. Una aplicación a las empresas de servicios profesionales de España. Cuadernos de Economía y Dirección de la Empresa, (40), pp. 83-109. https://doi.org/10.1016/S1138-5758(09)70043-X

Meljem, S., Revah, B., Cruz, Y. y Romero N. (2010). Valuación y Reconocimiento de Activos Intangibles. Un enfoque estratégico. México: IMEF, Price Waterhouse Coopers e ITAM.

Merritt, H. (2012). Las empresas mexicanas de base tecnológica y sus capacidades de innovación: una propuesta metodológica. Trayectorias, Año 14, Núm. 33-34, Julio 2011-junio 2012, 27-50. 
Motta, J. J., Zavaleta, L., Llinás, I., y Luque, L. (2013). Procesos de innovación y competencias de los recursos humanos en la industria del software en Argentina. Revista Iberoamericana de Ciencia, Tecnología y Sociedad, 24(8), 145-145.

Nonaka, I. (2007). La empresa creadora de conocimiento. Harvard Business Review. Julio 2007. Disponible https:// bschogardecristo.files.wordpress.com/2007/08/nonaka_red.pdf

Naranjo, C., Rubio, J., Salazar, L., Robledo, A.., \& Duque, J. (2013). Indicadores de capital intelectual. Memorias, 11(19), 39-51.

Nevado, D. y López, V. (2013). Gestione y controle el valor integral de su empresa. Análisis integral: modelos, informes financieros y capital intelectual para rediseñar la estrategia. Madrid: Ediciones Díaz de Santo.

Nevado, D. y López, V. (2004). Una herramienta de gestión de intangibles ocultos mediante técnicas econométricas. Método y aplicación. Revista Contabilidad y Dirección, 1, pp. 163-182.

OCDE (2005) Oslo Manual: Guidelines for Collecting and Interpreting Innovation, 3rd Edition. OECD Publications, Paris.

Ochoa, M., Prieto, M. y Santidrián, A. (2010) Estado actual de los modelos de capital intelectual y su impacto en la creación de valor en empresas de Castilla y León. España: Consejo Económico y Social de Castilla y León.

Ospina, S. T., Berrío, F. G., Bedoya, I. B., Cárdenas, M. V., y Muñoz, J. E. (2013). Medición de capital intelectual usando estadística multivariada. Revista Ingeniería Industrial, 1(1), 77-84.

Ordoñez De Pablos, P. (2004). El capital estructural organizativo como fuente de competitividad empresarial: un estudio de indicadores. España. Economía industrial, (357), Pp.131-140.

Padilla, L. (2015). Barreras para la innovación en las pymes colombianas de base tecnológica: una mirada desde la gestión. Universidad Militar Nueva Granada.

Ringle, C., Wende, S, y Will, A. (2005). SmartPLS 2.0.M3. Hamburg: SmartPLS. Retrieved from http://www. smartpls.com

Román, R., Gómez, A. y Simida, A. (2013). El capital social organizacional de la pequeña empresa innovadora. Un ensayo de medición en las ciudades de Cali y Medellín. Estudios Gerenciales, 29, p.356-367. https://doi. org/10.1016/j.estger.2013.09.010

Ross, J., et al (1997). Capital intelectual: el valor intangible de la empresa. Buenos Aires: Ediciones Paidós Ibérica.

Salazar, E. M. A. (2008). Capital intelectual y resultados empresariales en las empresas de servicios profesionales de España (Doctoral dissertation, Universidad Complutense de Madrid)

Sánchez, A. Melián, A. y Hormiga, E. (2007). El concepto del Capital Intelectual y sus Dimensiones. Investigaciones Europeas de Dirección y Economía de la Empresa, 13, (2), Pp. 97-111.

Santa, L. y Salas, M. (2014). El capital intelectual como esquema de valor en el futuro. Revista Estrategias, 10, (19), pp. 25-31. https://doi.org/10.16925/es.v10i19.518

Santos-Rodrigues, H., Dorrego, P. F., y Jardón, C. F. (2011). El capital estructural y la capacidad innovadora de la empresa. Investigaciones Europeas de Dirección y Economía de la Empresa, 17(3), 69-89. https://doi. org/10.1016/s1135-2523(12)60121-x

Sveiby, K. (2007). Methods for measuring intangible assets. Documento disponible en internet www.sveiby,com

Torres, L. (2012). La nueva dependencia: los activos intangibles. Capital intelectual, (15), pp. 14-35 